\title{
A Networked Approach for Assessing Risks to the Electric Grid
}

\author{
Patrick O’Connor ${ }^{1}$, Carl Dister ${ }^{1}$, Johnny Gest ${ }^{1}$, Tyson Browning ${ }^{2}$ \\ ${ }^{1}$ ReliabilityFirst Corporation \\ ${ }^{2}$ Neeley School of Business, Texas Christian University
}

\begin{abstract}
This paper examines the interrelatedness of risks in the context of risk analysis with the goal of providing valuable outcomes for regulators, specifically the example of ReliabilityFirst as a regulator of the bulk power system (BPS). A focus on the novel concept of the "time lag" associated with network risks demonstrates the importance of accurate risk ranking. Using a design structure matrix (DSM) to model relationships, the possibility of causal relationships between risks to the BPS was assessed by industry experts. The assessment asked the experts to identify the probability and impact of each risk in isolation, as well as a smaller subset of the experts weighting the interrelatedness of the identified risks. The outcomes show that applying a networked approach to risk analysis provides a more comprehensive understanding of risk relationships, which results in greater opportunities for addressing risks in the most effective order.
\end{abstract}

Keywords: risk management, risk dependencies, risk propagation, risk network, risk time lag

\section{Introduction}

The traditional concept of regulation involves a government authority ensuring that regulated organizations are in technical compliance with a system of rules and laws (Nicholls 2015). The more modern approach of risk-based regulation involves the regulator working collaboratively with the regulated organizations for the proactive prevention of harms. This requires the regulator to have a nimble, mission-driven approach to determine the issues it will focus on and utilize the appropriate mechanisms to influence behavior that will mitigate the potential harm posed by those issues. Sparrow (2011) described this process as the "craft of regulation."

ReliabilityFirst Corporation (RF) is one of six Regional Entities approved by the Federal Energy Regulatory Commission (FERC) to ensure the reliability, security, and resiliency of the North American bulk power system (BPS) pursuant to the Energy Policy Act of 2005. RF performs this mission pursuant to its delegation agreement with the North American Electric Reliability Corporation (NERC), which is the FERC-approved electric reliability organization. Considering the complex and interconnected nature of the BPS, the NERC, RF, and the other Regional Entities (collectively, the "ERO Enterprise") have adopted a risk-based approach to regulating the users, owners, and operators of the BPS.

Normally, RF has crafted its regulation through a classical, top-down approach to risk identification and assessment focused on the primary factors of probability and impact. 
These assessments consider the various risks in isolation, without accounting for their interrelatedness. Drawing upon risk network analysis research in the field of project management (e.g., Fang and Marle 2012), RF sought to take this next evolutionary step in its approach to risk assessments.

Project risk management (PRM) has matured to the point of acknowledging the high level of complexity inherent in projects today. The existence of numerous and diverse elements which are strongly interrelated is one of the main features of this complexity. Academic research has highlighted the network of interdependent risks accompanying projects and even sought to examine the propagation behavior of these risks resulting in a "domino effect" or a "loop."

In this paper, RF seeks to build upon the extensive work on risk network analysis from the project management field by applying those same concepts to the risks facing the BPS. One innovative outcome of this endeavor is the concept of the "time lag" associated with a risk in a network. In the BPS context, a risk may manifest itself in Year One, and its effects may linger on the grid for years, amplifying the likelihood of other risks. To account for this time lag, RF developed an algorithm based upon the Interpretive Structural Modeling (ISM) approach described by Gorvett and Liu (2007).

The purpose of developing a networked approach to risk analysis is to provide the ERO Enterprise and any other risk-based regulator with a decision support system framework based on a more complete picture of the risks they are attempting to manage, while understanding that the essence of the craft of regulation is action with neither entire ignorance nor complete and perfect information, but partial knowledge.

\section{Epistemological Approach to Risk Assessment}

One of the challenges present in the craft of regulation is that human perception and cognition are inherently limited (Renn 2014). Consequently, it is often impossible to generate perfect knowledge about our world or create a "true" understanding of our physical and social environments. This type of epistemological problem creeps into the realm of risk analysis in different ways. It can be demonstrated in questions like "How do we know the real risks we face?" or "How can we rank these risks objectively?" This epistemological quagmire requires risk-based regulators to sift through these competing epistemologies to develop a risk assessment methodology that is both sound and fair. RF acknowledges that all of its risk assessments are influenced by the assessors' own background and experience. In this and all assessments, RF takes steps to reduce the potential of bias on the part of the assessors.

\section{Expert Elicitation}

Expertise in the ERO Enterprise can generally be separated into two main domains: power systems engineering and cybersecurity. For this exercise, subject matter experts (SMEs) were chosen from both domains based upon their educational backgrounds and professional experience. To control for overconfidence bias, all SMEs had undergone calibration training as described by Hubbard (2009). All of the SMEs participated in the classical risk assessment analysis by independently completing a survey that asked them 
to identify the probability and impact of each identified risk in isolation. A smaller subset of four SMEs, two power systems engineering SMEs and two cybersecurity SMEs, were selected to participate in the expert elicitation for the network analysis.

Following the guidance provided by the U.S. Nuclear Regulatory Commission in its 2016 white paper on expert elicitation (Xing and Morrow 2016), this subgroup of SMEs was tasked with identifying and weighting the interrelatedness of the identified risks using a design structure matrix (DSM)-based method. The subgroup SMEs were selected based on their experience utilizing DSMs and the fact that they worked in a RF department not focused strictly on compliance. Rather, these individuals' primary work involved a high degree of cross-functional work that exposed them to many different perspectives on the risks facing the BPS.

The elicitation session to build the risk structure matrix (RSM) lasted approximately two hours. The SMEs were asked the following question for each pair of risks in the network: "If Risk A occurs in Year One, how likely is it that this occurrence could cause Risk B to occur in the following year?" The various weights placed on the connections among risks (i.e., 0, 1, 3,9) accounted for the time-lag aspect of the risks. The following example helps illustrate this relationship. An owner of assets on the BPS implements a system reinforcement in Year One to enhance overall performance and reliability. During the final testing of the control systems associated with this system reinforcement, certain tests were either performed erroneously or not at all. As a result, a latent error resides within the function of this particular control system on the BPS, which could cause an inadvertent interruption of service later. Therefore, if a latent error is developed in Year One, it may be more likely that an inadvertent interruption of service could occur in Year Two when the control system reacts to an event like a lightning strike. Based on the aforementioned weights, the time-lag aspect of the risks in this example are as follows: a value of 9 would indicate an interruption of service has a strong likelihood to occur in Year 2; a value of 3 has a moderate chance to occur, a value of 1 has a slight chance to occur, and a value of 0 has no chance to occur.

\section{Risk Identification and Initial Assessment}

RF began with a list of conventional risks with negative effects, followed by a classical assessment of these risks in terms of probability and impact. As suggested by Hubbard (2009), this exercise employed the subjectivist perspective on probability. First, the experts on the RF team were asked to identify the potential level of harm (impact) for each risk category. In accordance with the NERC's Event Analysis Process (NERC 2019), the scales in Tables 1 and 2 were used for responses. Experts assessed the probability of a risk occurring in a given year and the impact in terms of the number of megawatts that would be lost. The classical steps of risk identification and assessment study individual risks in isolation. These results, given in Table 3, serve as an input to our further study of risk interactions. 
Table 1. Impact Table

\begin{tabular}{|c|l|}
\hline Statement & \multicolumn{2}{|c|}{ Impact } \\
\hline Extreme & $\begin{array}{l}\text { Unintended loss of load and/or } \\
\text { generation }>10,000 \mathrm{MWs}\end{array}$ \\
\hline Substantial & $\begin{array}{l}\text { Unintended loss of load and/or } \\
\text { generation from 5,000-10,000 MWs }\end{array}$ \\
\hline Intermediate & $\begin{array}{l}\text { Unintended loss of load and/or } \\
\text { generation from 1,000-4,999 MWs }\end{array}$ \\
\hline Minor & $\begin{array}{l}\text { Unintended loss of load and/or } \\
\text { generation from 300-999 MWs }\end{array}$ \\
\hline None & $\begin{array}{l}\text { Unintended loss of load and/or } \\
\text { generation < 300 MWs }\end{array}$ \\
\hline
\end{tabular}

Table 2. Likelihood Table

\begin{tabular}{|l|r|}
\hline \multicolumn{1}{|c|}{ Statement } & \multicolumn{1}{c|}{ Likelihood } \\
\hline Extremely Likely & 1 \\
\hline Very Likely & 0.33 \\
\hline Likely & 0.125 \\
\hline More Moderate & 0.05 \\
\hline Moderate & 0.0125 \\
\hline Less Moderate & 0.0025 \\
\hline Unlikely & 0.001 \\
\hline Very Unlikely & 0.00001 \\
\hline Extremely Unlikely & 0.000001 \\
\hline Not Likely & 0.0000001 \\
\hline
\end{tabular}

Table 3. Grid Risks with Probability and Impact in Isolation

\begin{tabular}{|c|c|c|c|c|}
\hline Risk Category & Abbreviation & $\begin{array}{c}\text { Likelihood in } \\
\text { Isolation } \\
\rho_{i}\end{array}$ & $\begin{array}{c}\text { Impact in MW in } \\
\text { Isolation } \\
\theta_{i}\end{array}$ & $\begin{array}{c}\text { Expected Loss } \\
\text { (Likelihood X } \\
\text { MW) }\end{array}$ \\
$\rho_{i} \theta_{i}$ \\
\hline Xrotection System Misoperations & MIS & 0.19 & 6824 & 1327 \\
\hline Information/Asset Security & CYP & 0.11 & 8235 & 905 \\
\hline Environmental Factors & ENV & 0.10 & 8000 & 768 \\
\hline Human Performance & HPF & 0.10 & 6737 & 643 \\
\hline Changing Generation Mix & GEN & 0.06 & 5800 & 359 \\
\hline Situational Awareness and IROLS & SAW & 0.04 & 6778 & 260 \\
\hline Event Response & EVR & 0.04 & 6800 & 246 \\
\hline Unknown Unknowns & UNK & 0.03 & 7200 & 230 \\
\hline Planning and Modeling & MPL & 0.02 & 6000 & 132 \\
\hline
\end{tabular}

\section{Risk Network Model}

\subsection{Identification of Risk Network}

The DSM method (Eppinger and Browning 2012) has proven to be a practical tool for representing and analyzing relations and dependencies among system components. The DSM is used here to model the relationships among grid risks, since one risk may influence another. Risk interaction is considered as the existence of a possible causal relationship between two grid risks. The risk structure matrix (RSM) is defined as a DSM with RSM $\mathrm{ij}_{\mathrm{j}}$ $=\{0,1,3,9\}$ representing a $\{$ no, slight, moderate, strong $\}$ link from $R_{j}$ to $R_{i}$ (i.e., inputsin-rows [IR] DSM convention). Figure 1 gives an example where an empty cell indicates a 0 and darker shading indicates stronger links.

As mentioned, the experts also encoded a time dependency into the RSM (i.e., 0, 1, 3, and 9) which have the following assigned likelihoods, respectively: 0, 0.11, 0.33, and 1.0. To account for time-lag effects, any direct link (greater than zero) was assumed to cause a 50\% increase in the probability of all second-order, indirectly affected risks in the following 
year. ${ }^{1}$ (This amplified probability is then negligible after the following year.) For example, in Figure 1, an environmental risk (ENV) occurring now could directly cause a generation risk (GEN) this year and could furthermore cause (50\% likelihood) a misoperation risk (MIS) next year. These indirect effects are layered on top of the direct effects to account for the increased risk due to the indirect relationships and time lags.

Figure 1. Illustration of the risk structure matrix (RSM) and equivalent directed graph
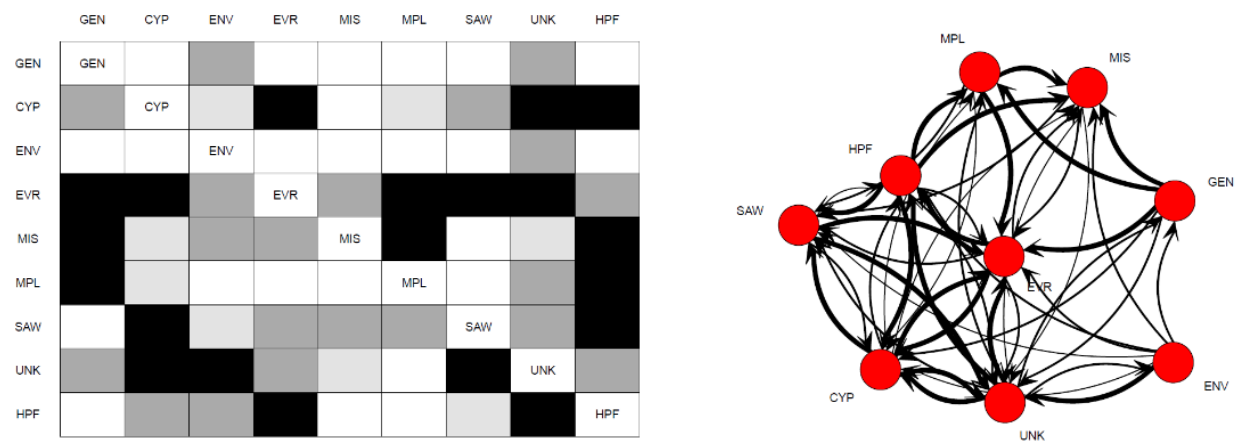

Figure 2. Description of the transformation from isolated to networked risks using the RSM

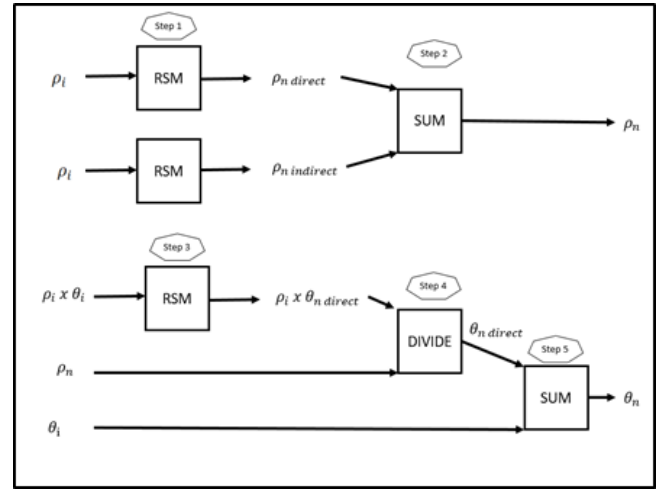

\subsection{Assessing the Risk Network}

The risks initially evaluated as listed in Table 3 were then subjected to a five-step process (outlined in Figure 2) to account for their interactions.

1. Calculate networked probabilities from direct links: For each risk, calculate its larger, networked probability using the RSM for all direct connections. For example, an environmental risk (ENV) has a $10 \%$ likelihood of occurring. A generation risk (GEN) has a $6 \%$ likelihood. Since an ENV risk is connected to a GEN risk by a weight of 3 , the

\footnotetext{
${ }^{1}$ This $50 \%$ factor was the result of discussion with the experts, who found the number to be reasonable and realistic. Furthermore, based on the sensitivity analyses, described in Section 6 below, reasonable changes to this factor did not make a significant difference in the results.
} 
likelihood of the GEN risk increases from $6 \%$ to $6 \%+(1 / 3$ of $10 \%)$ or $9.33 \%$ from just the ENV risk.

2. Calculate networked probabilities from indirect links: Like in Step 1, calculate the larger, networked probability using the RSM for all indirect connections. The contributions from direct connections and indirect connections (one year prior) are added to estimate the final networked probability. For example, an ENV risk has a $10 \%$ likelihood of occurring. A GEN risk has a $6 \%$ likelihood. The GEN risk is connected to an ENV risk by a weight of 3 . The GEN risk then connected to the protection system mis-operations (MIS) risk, which has a 19\% likelihood. This creates an indirect connection between ENV and MIS. The original likelihood of MIS will increase due to this indirect connection from $19 \%$ to $19 \%+6 \%$ (direct from GEN) $+50 \%$ of $10 \%$ indirect from ENV) or $30 \%$ total for the MIS risk.

3. Calculate expected losses: The amplified probability and impact are used to revise the expected losses, but only for the direct connections. (The impact from a prior year is not counted in a current year.) For example, an ENV risk has an impact of 8,000 MW. A GEN risk has a 5,800 MW impact. Since an ENV risk is connected to a GEN risk by a weight of 3, the potential impact of the GEN risk increases from 5,800 MW to 5,800 $\mathrm{MW}+(1 / 3$ of $8,000 \mathrm{MW})$ or 8,476 MW from just the ENV risk.

4. Calculate the networked impact: The networked impact in the current year from all direct effects is calculated by dividing by the networked probabilities from step $2{ }^{2}$

5. Calculate the networked harm: The networked harm is finally determined by adding the harm in isolation to the additional harm created by direct connections (although at lower probabilities of occurrence) ${ }^{2}$

The equations governing these steps are described as follows:

$\rho_{i}=$ Likelihood of the Risk Occuring in Isolation ( 0 to 1 )

$\theta_{i}=$ Impact if the Risk Occured (0 to 10,000 Megawatts Load Loss)

$\rho_{n}=$ Likelihood of the Risk Occuring with Interconnectedness ( 0 to 1$)$

$\theta_{n}=$ Impact if the Risk Occured (0 to 10,000 Megawatts Load Loss)

$\rho_{n}=\rho_{i}+\left[\sum_{\text {connections }}^{\text {all direct }} \frac{\alpha_{\text {direct }}}{9} \times \rho_{\text {idirect }}\right]+\frac{1}{2} \times\left[\sum_{\text {connections }}^{\text {allindirect }} \frac{\alpha_{\text {idirect }}}{9} \times \rho_{\text {i indirect }}\right]$

Where $\alpha_{\text {direct or indirect }}$ is the weight listed in the RSM: $0,1,3$ or 9 . Note that $\rho_{n} \geq \rho_{i}$. In other words, the risk always increases and never decreases as a result of the network effects. The networked risk is then given by:

$\rho_{n} \times \theta_{n}=\rho_{i} \times \theta_{i}+\left[\sum_{\text {connections }}^{\text {all direct }} \frac{\alpha_{\text {direct }}}{9} \times \rho_{\text {i direct }} \times \theta_{\text {direct }}\right]+\frac{1}{2} \times\left[\sum_{\text {all indirect }}^{\text {connections }} \frac{\alpha_{\text {idirect }}}{9} \times \rho_{i \text { indirect }} \times\right.$ $\left.\theta_{\text {indirect }}\right]$

The initial and final likelihoods and impacts are plotted in Figure 3, with the tip of each arrow representing the networked values. In all cases, the risk links served to substantially increase the expected losses (Table 1). Each risk in isolation (indicated by the beginning of the arrow) increased to a new level after accounting for network influences (indicated

${ }^{2}$ Considering the fact that the entire network is used in this calculation, examples would be too lengthy here. Please see the software equations in the Appendix. 
by the tip of the arrow). The EVR risk increased significantly, for example, while ENV risk did not change as dramatically.

Figure 3. Shifts in Risk Ranking

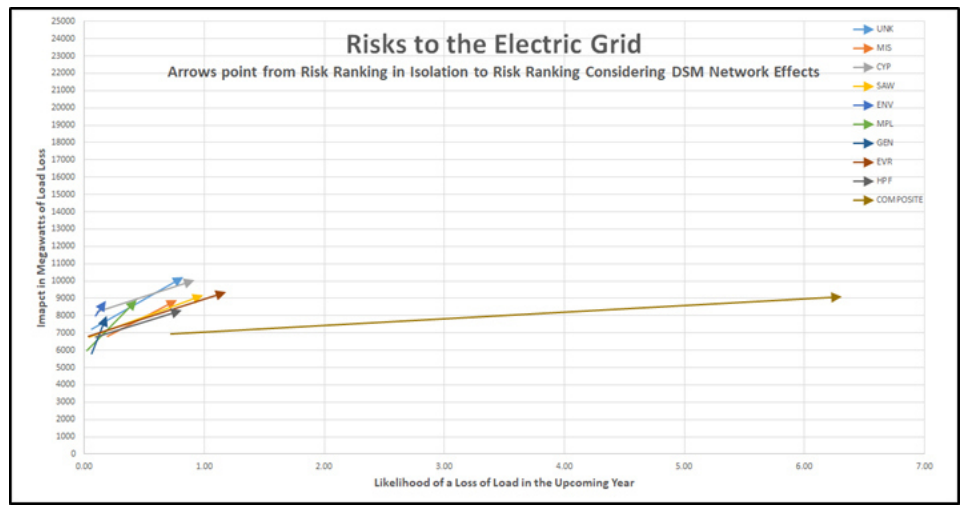

Figure 4. Comparison of Final Rankings

\begin{tabular}{|l|r|}
\hline \multicolumn{2}{|c|}{ Expected Loss in Isolation } \\
\hline Risk Category & \multicolumn{1}{|c|}{$\rho_{\mathrm{l}} \times \theta_{\mathrm{l}}$} \\
\hline MIS & 1327 \\
\hline CYP & 905 \\
\hline ENV & 768 \\
\hline HPF & 643 \\
\hline GEN & 359 \\
\hline SAW & 260 \\
\hline EVR & 246 \\
\hline UNK & 230 \\
\hline MPL & 132 \\
\hline
\end{tabular}

\begin{tabular}{|l|r|}
\hline \multicolumn{2}{|c|}{ Expeced Loss Networked } \\
\hline Risk Category & $\rho_{\eta} \times \theta_{\eta}$ \\
\hline EVR & 11087 \\
\hline CYP & 9232 \\
\hline SAW & 9061 \\
\hline UNK & 8464 \\
\hline MIS & 6897 \\
\hline HPF & 6768 \\
\hline MPL & 3861 \\
\hline ENV & 1607 \\
\hline GEN & 1484 \\
\hline
\end{tabular}

\section{Sensitivity Analyses}

Utilizing the RSM not only allows the consideration of complex relationships between risk categories when determining the overall risk rank but also provides additional insight in the development of a risk mitigation plan by factoring in how sensitive any risk's ranking is to changes in model parameters. As the effectiveness of risk mitigation activities are monitored over time, further perspective can be gained by validating and/or updating the initial uncertainty within the original RSM. Initially, a sensitivity analysis also can be performed to determine which risk links should be monitored most closely. The sensitivity of the link weights (i.e., 1, 3, and 9) were studied first to determine their impact on the risk ranking. In addition to the original networked analysis, three additional scenarios were considered: 1) changing slight links to strong ones $\left(\mathrm{RSM}_{\mathrm{ij}}=1\right.$ changed to 9$\left.) ; 2\right)$ changing a moderate link to a strong one $\left(\mathrm{RSM}_{\mathrm{ij}}=3\right.$ changed to 9$)$; and 3$)$ adjusting both slight and moderate links to strong ones $\left(\mathrm{RSM}_{\mathrm{ij}}=1\right.$ and 3 changed to 9$)$. Table 4 displays variances in likelihood, impact, risk, and rank for each of these scenarios. 
Table 4. Risk Linkage Strength - Sensitivity Review

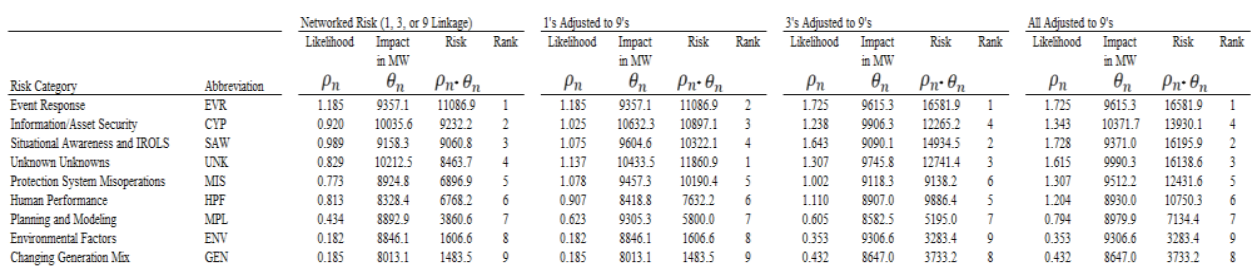

This analysis resulted in values associated with likelihood, impact, and risk changing significantly for multiple risks. Figure 5 shows the variations, with UNK and SAW being the most sensitive risk categories (with the greatest amounts of variation).

Figure 5. Risk Variation in Link Strength Sensitivity Review

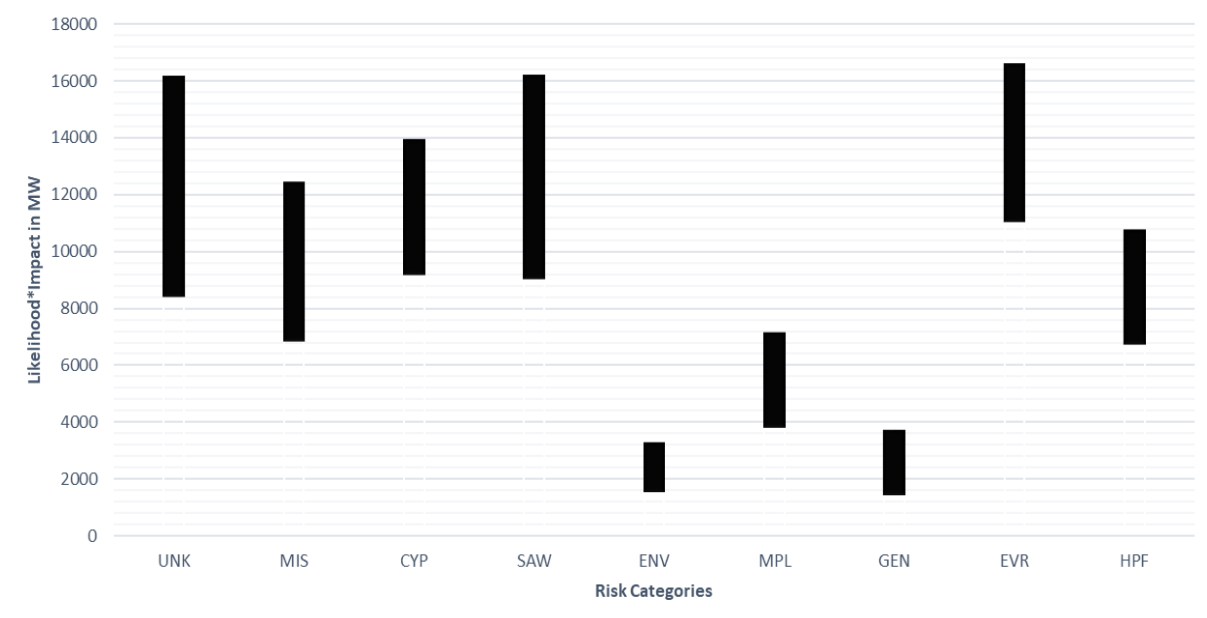

However, Table 4 indicates that the overall risk rankings did not change significantly with changes in link strength. In fact, EVR, CYP, SAW, and UNK consistently remained the top four risk categories. The remaining risk categories also stayed relatively consistent with no movement in rank or only moving one position. Second, we analyzed the sensitivity of the results to changes in the probabilities (likelihoods). In short, the model was rerun with each risk's likelihood cut in half. This analysis indicated that a reduction in the likelihood of CYP has the largest effect on the top four risk categories. As a result, risk mitigation activities should initially focus on CYP initiatives.

\section{Conclusion}

This paper presented a networked approach for assessing the risks to the BES in the RF footprint. This approach supplements the current approach, which only focuses on assessing risks in isolation. Notably, the approach detailed in this paper also adds the timelag aspect of these risks, which refers to the fact that a risk occurring in Year One may linger on the grid, amplifying the likelihood of other risks occurring in Year Two. These 
results indicate that utilizing this networked approach has an impact on the order in which the risks are ranked (Figure 4). These results also provide a different perspective on RF's focus of risk mitigation activities. While there are certainly limitations to this approach, it nevertheless provides RF and the ERO with a more complete picture of the risks facing the grid today. Future collaboration with regulators of other critical infrastructures based on this approach can expand the network of risks by linking risks between infrastructures.

\section{References}

Eppinger, S.D. and T.R. Browning (2012) Design Structure Matrix Methods and Applications, Cambridge, MA: MIT Press.

Fang, C. and F. Marle (2012) "A Simulation-based Risk Network Model for Decision Support in Project Risk Management," Decision Support Systems, 52(3): 635-644.

Gorvett, R. and Liu, N. (2007), "Measuring Operational Risk Interdependencies using Interpretive Structural Modeling," 2007 Enterprise Risk Management Symposium, Chicago, IL.

Hubbard, D. (2009) The Failure of Risk Management, Hoboken, NJ: Wiley.

NERC (2019), "Electric Reliability Organization Event Anlaysis Process Version 4.0," available at: https://www.nerc.com/pa/rrm/ea/ERO_EAP_Documents\%20DL/ERO_EAP_v4.0_final.pdf

Nicholls, A. (2015) "The Challenges and Benefits of Risk-Based Regulation in Achieving Scheme Outcomes," Presented to the Actuaries Institute, Injury Schemes Seminar, 8-10 November.

Renn, O. (2014). The Risk Society Revisited, Temple University Press.

Sparrow, M. (2011) The Regulatory Craft. Washington, DC: Brookings Institute Press.

Xing, J. and S. Morrow (2016), "White Paper: Practical Insights and Lessons Learned on Implementing Expert Elicitation," available at: https://www.nrc.gov/docs/ML16287A734.pdf

Contact: Patrick O'Connor, ReliabilityFirst Corporation, 3 Summit Park Drive, Suite 600, Cleveland, OH, United States, 216-503-0668, patrick.oconnor@ rfirst.org

\footnotetext{
Appendix: Software

The spreadsheet Risk_Analysis_Software.xls contains the equations to calculate the Likelihood of Occurrences and Impacts. There are sensitivity analysis tuning parameters included - e.g., the "one half" concept from the experts is tunable. Also, the weighting of 1,3 , or 9 is adjustable to check for sensitivity between uncertainties in the experts' expression of the connection weight and its impact on the final ranking.

R Software

The following R commands were run to create the network diagrams and the list of direct and indirect connections for use in the spreadsheet. The package "sna" was used to create the graphics (Note: It could have been assumed that every cell in the DSM was full and loops run to calculate every combination; however, the sparse matrix was leveraged, along with the small number of risks, to allow a more semi-automatic coding, using spreadsheets that non-programmers could understand and verify/validate.

$\operatorname{library}($ sna $)$

A9=matrix $(\mathrm{c}(0,0,0,1,1,1,0,0,0,0,0,0,1,0,0,1,1,0,0,0,0,0,0,0,0,1,0,0,1,0,0,0,0,0,0,1,0,0,0,0,0,0,0,0,0,0,0,0,1,1,0,0,0,0,0,0,0,1,0,0,0,1,0,0,1,0,1,0,0,0,0,1$, $0,1,0,0,1,1,1,0,0)$, nrow $=9$, ncol=9, byrow=FALSE)

$\mathrm{A} 3=$ matrix $(\mathrm{c}(0,1,0,0,0,0,0,1,0,0,0,0,0,0,0,0,0,1,1,0,0,1,1,0,0,0,1,0,0,0,0,1,0,1,1,0,0,0,0,1,0,0,1,0,0,0,0,0,0,0,0,1,0,0,0,1,0,0,0,0,0,0,0,1,0,1,0,0,1,1,0,0$, $0,0,0,1,0,0,0,1,0)$, nrow $=9$, ncol $=9$, byrow=FALSE)

$\mathrm{A} 1=$ matrix $(\mathrm{c}(0,0,0,0,0,0,0,0,0,0,0,0,0,1,1,0,0,0,0,1,0,0,0,0,1,0,0,0,0,0,0,0,0,0,0,0,0,0,0,0,0,0,0,1,0,0,1,0,0,0,0,0,0,0,0,0,0,0,0,0,0,0,1,0,0,0,0,1,0,0,0,0$, $0,0,0,0,0,0,0,0,0)$, nrow $=9$, ncol $=9$, byrow $=$ FALSE)

AT $=$ matrix $(\mathrm{c}(0,3,0,9,9,9,0,3,0,0,0,0,9,1,1,9,9,3,3,1,0,3,3,0,1,9,3,0,9,0,0,3,0,3,3,9,0,0,0,3,0,0,3,1,0,0,1,0,9,9,0,3,0,0,0,3,0,9,0,0,0,9,1,3,9,3,9,1,3,3,0,9$,

$0,9,0,3,9,9,9,3,0)$,nrow $=9$, ncol=9, byrow $=$ FALSE)

\#calculate the reachability matrices

A $92<-\mathrm{A} 9 \% * \% \mathrm{~A} 9$

$\mathrm{A} 32<-\mathrm{A} 3 \% * \% \mathrm{~A} 3$

$\mathrm{A} 12<-\mathrm{A} 1 \% * \% \mathrm{~A} 1$

\#AT2<-AT\%*\%AT

\# Make the labels

labeldata<-c("GEN","CYP","ENV","EVR","MIS","MPL","SAW","UNK","HPF")
} 
datalabel=data.frame(row=c("GEN","CYP","ENV","EVR","MIS","MPL","SAW","UNK","HPF"),column=c("GEN","CYP","ENV","EVR","MIS"," MPL","SAW","UNK","HPF"))

\#Create the plots and DSMs

A9TR<-t(A9)

gplot(A9TR,usecurv=TRUE,edge.lwd=0.5,vertex.cex $=2$, label=labeldata)

sociomatrixplot $(\mathrm{A} 9$,labels=datalabel $)$

sociomatrixplot $(\mathrm{A} 92$, labels $=$ datalabel $)$

A3TR<-t(A3)

gplot(A3TR,usecurv=TRUE, edge. $1 w d=0.5$, vertex.cex $=2$, label=labeldata)

sociomatrixplot $(\mathrm{A} 3$, labels $=$ datalabel $)$

sociomatrixplot $(\mathrm{A} 32$, labels $=$ datalabel $)$

A1TR<-t(A1)

gplot(A1TR,usecurv=TRUE,edge.lwd=0.5,vertex.cex $=2$,label=labeldata)

sociomatrixplot $(\mathrm{A} 1$, labels $=$ datalabel $)$

sociomatrixplot $(\mathrm{A} 12$, labels $=$ datalabel $)$

ATR<-t(AT)

gplot(ATR,usecurv=TRUE,edge.lwd $=0.5$,vertex.cex $=2$, label=labeldata)

sociomatrixplot (AT,labels=datalabel)

sociomatrixplot(AT2,labels=datalabel)

\# unused functions

$\# \mathrm{r}=$ reachability $(\mathrm{A})$

\#geodist(A) \#see counts

\#prestige(A) \#opposite of

\#neighborhood(A,1) \#shows the first sets by column

\#neighborhood(A,2) \#show the second sets by column

\#Convert to igraphs and produce the edge lists - paste these into Excel - look at chart to find middles for the seconds

library(igraph)

g<-graph_from_adjacency_matrix(A9TR, mode =c("directed"), weighted = NULL,diag = FALSE, add.colnames = labeldata, add.rownames =

labeldata)

vertex $\operatorname{attr}(\mathrm{g})<-$ list $($ name $=$ labeldata $)$

as_edgelist $(\mathrm{g})$

A92TR<-t(A92)

g<-graph_from_adjacency_matrix $($ A92TR, mode $=\mathrm{c}($ "directed"), weighted $=$ NULL,diag $=$ FALSE, add.colnames = labeldata, add.rownames $=$ labeldata)

vertex_attr $(\mathrm{g})<-$ list $($ name $=$ labeldata)

as_edgelist $(\mathrm{g})$

g<-graph_from_adjacency_matrix $($ A3TR, mode $=\mathrm{c}($ "directed"), weighted = NULL,diag $=$ FALSE, add.colnames = labeldata, add.rownames $=$ labeldata)

vertex_attr $(\mathrm{g})<-$ list $($ name $=$ labeldata $)$

as_edgelist $(\mathrm{g})$

A32TR<-t(A32)

g<-graph_from_adjacency_matrix $($ A32TR, mode $=\mathrm{c}($ "directed"), weighted $=$ NULL,diag $=$ FALSE, add.colnames = labeldata, add.rownames $=$ labeldata)

vertex_attr $(\mathrm{g})<-$ list $($ name $=$ labeldata)

as_edgelist $(\mathrm{g})$

g<-graph_from_adjacency_matrix $($ A1TR, mode $=\mathrm{c}($ "directed"), weighted $=$ NULL,diag $=$ FALSE, add.colnames $=$ labeldata, add.rownames $=$ labeldata)

vertex_attr $(\mathrm{g})<-$ list $($ name $=$ labeldata $)$

as edgelist $(\mathrm{g})$

A12TR<-t(A12)

g<-graph_from_adjacency_matrix $($ A12TR, mode $=\mathrm{c}($ "directed"), weighted = NULL,diag = FALSE, add.colnames = labeldata, add.rownames $=$ labeldata)

vertex_attr $(g)<-$ list $($ name $=$ labeldata)

as_edgelist $(\mathrm{g})$

g<-graph_from_adjacency_matrix $($ ATTR, mode $=\mathrm{c}($ "directed"), weighted $=$ NULL,diag $=$ FALSE, add.colnames $=$ labeldata, add.rownames $=$ labeldata)

vertex_attr $(\mathrm{g})<-$ list $($ name $=$ labeldata)

as edgelist $(\mathrm{g})$

AT2TR<-t(AT2)

$\mathrm{g}<$-graph_from_adjacency_matrix $($ AT2TR, mode $=\mathrm{c}($ "directed"), weighted $=$ NULL,diag $=$ FALSE, add.colnames $=$ labeldata, add.rownames $=$ labeldata)

vertex_attr(g) <- list(name = labeldata)

as_edgelist $(\mathrm{g})$ 